\title{
ISO 9001 version 2015 implementation guide
}

\author{
ISO 9001 versión 2015 guía de implementación revisión de caso
}

\author{
Á. M. Buriticá-Macías ; C. A. Buriticá-Noreña ; G. López-Quintero iD
}

\begin{abstract}
Companies that require the implementation of the new version of the quality standard ISO 9001: 2015, must go to a quality consultant to make their application, implying costs and more time to meet this objective. Through this implementation guide, organizations are facilitated to apply the requirements without the need of an advisor and adapt it to their needs. A revision was made to the new version and to the previous one where modifications were found to the numerals from 8 to 10 and structurally emphasized in the context of the organization by including the internal and external factors that affect it. This allows to comply with the requirements of the client in a coherent manner, legal and regulatory in order to increase the satisfaction of the same; it also involved the risks as an effect of an uncertainty associated with its context and the objectives of the organization and incorporated them into the requirements of the standard. Finally, the changes with respect to the 2008-year version are presented so that they can be applied in the update to the new version in the companies that require it.
\end{abstract}

Index terms- Certification, Continuous improvement, Management system, planning, procedure, strategic direction requirements, Risk, verification, requirements.

Resumen-Las empresas que requieran la implementación de la nueva versión de la norma de calidad ISO 9001:2015, deben acudir a un asesor de calidad para realizar su aplicación, implicando costos y mayor tiempo para cumplir con este objetivo. Mediante esta guía de implementación se facilita a las organizaciones aplicar los requerimientos sin necesidad de un asesor y adaptarla a sus necesidades. Se realizó una revisión a la nueva versión y a la anterior donde se encontraron modificaciones a los numerales de 8 a 10 y estructuralmente se dio énfasis en el contexto de la organización al incluir los factores internos y externos que la afectan. Esto permite dar cumplimiento de manera coherente a los requisitos del cliente, los legales y reglamentarios con el propósito de aumentar la satisfacción del mismo; también involucró los riesgos como efecto de una incertidumbre asociados a su contexto y a los objetivos de la organización y los incorporó a los requisitos de la norma. Finalmente se presentan los cambios respecto a la versión año 2008 para que sean aplicados en la actualización a la nueva versión en las empresas que lo requieran.

Palabras claves - Certificación, dirección estratégica, mejora continua, planeación, procedimiento, proceso, requerimientos. riesgo, sistema de gestión.

Este manuscrito fue enviado el 15 de mayo de 2018 y fue aceptado el 29 de marzo de 2019.

Á. M. Buritica-Macias, Ingeniería Industrial Universidad Tecnológica de Pereira, Colombia, (amburitica@utp.edu.co).
I. INTRODUCCION

T A adopción de un sistema de gestión de la calidad es una Ldecisión estratégica, que depende de tipo y tamaño de la organización, entre más complejo sea el sistema se espera que ayude a mejorar su desempeño, a cumplir los objetivos específicos con las necesidades y expectativas de los clientes y a desarrollar sus procesos buscando la oportunidad de reducir o eliminar los riesgos, para alcanzar lo propuesto en la norma, Podemos utilizar esta guía de implementación que permitirá a las organizaciones ahorrar en los costos iniciales de un asesor y sirve para actualizar las norma de versión 2008 o documentar la nueva versión de la ISO 2015, la entidad nacional de acreditación de España ENAC publico una guía de implementación en el año 2008 de la norma en la WEB, pero la ISO obligo a retirarla, motivo por el cual en este artículo elaboramos una guía técnica que ayuda a las empresas a elaborar la documentación, a través del estudio de referencias normativas y aplicaciones de la norma en empresas se encuentra como conclusión principal que la filosofía de la nueva versión está fundamentada en el pensamiento basado en el riesgo y su identificación para cumplir con los objetivos de la empresa.

\section{MetOdologíA}

\section{A. Objeto y campo de aplicación}

Todos los requisitos son aplicables a todo tipo de organizaciones, sean de servicio o que producen bienes materiales; sin importar el tamaño se debe especificar qué es lo que se quiere certificar en el campo de aplicación de la institución [1].

\section{B. Referencias normativas}

De acuerdo con la nueva versión no se tienen referencias normativas, pero si es compatible con otros sistemas de gestión y cada organización las puede utilizar como estime conveniente, como es la ISO 31000 de gestión del riesgo, la ISO 14000 de gestión ambiental etc.

C. A. Buriticá-Noreña., Ingeniería Industrial Universidad Tecnológica de Pereira, Pereira Colombia (caburitica@utp.edu.co).

G. López-Quintero, Ingeniería Industrial Universidad Tecnológica de Pereira, Pereira Colombia (jlq2012@utp.edu.co) 


\section{Términos y definiciones}

En esta versión la norma tiene 69 definiciones [2], que además hacen parte de la norma ISO 9000, e incorpora los términos relacionados con los riesgos; estas definiciones son un diccionario de calidad y pueden ser consultadas cuando sean requeridas.

\section{Contexto de la organización}

En este requisito se le dio más importancia a la dirección estratégica y al peso que toma en toda la norma; la misión de la organización debe tener una relación con la política de la calidad y su visión con los objetivos al corto, mediano y largo plazo deben ser coherentes con el negocio; es nuevo en las partes que tiene que ver con la identificación de la situaciones internas y externas que afectan las capacidades para alcanzar los resultados planificados por la organización en su sistema de calidad; se evidencia realizando la identificación de las debilidades y amenazas y considerando los entornos legales, económicos, culturales, sociales, tecnológicos, de mercado , competitivo etc. Sean internacionales o nacionales que no permiten lograr los objetivos, todo lo que pueda afectar es el insumo de la oportunidad de mejora para tratarlo en el numeral 6 de esta versión, la comprensión de las necesidades y expectativas de los empleados, accionistas, proveedores, clientes, y el entorno de la institución identificando sus requisitos de la manera como pueden afectar el sistema de gestión de la calidad.

Para determinar el alcance del sistema de gestión de la calidad, la organización especifica sus productos y servicios, los requisitos de las partes interesadas; si uno o varios de los requisitos la institución no los efectúa, cada uno debe justificar que no se aplican

La caracterización de los procesos y su mapa de procesos continúan como en la anterior versión, agregándole la identificación de los riesgos [3] que se puedan presentar en ellos y determinar las oportunidades de mejora; se hicieron más explícitos los componentes que se deben colocar en la caracterización, como son los elementos de entrada y de salida, los recursos que se requieren, los indicadores, la asignación de las responsabilidades y autoridad.

\section{E. Liderazgo}

Como en la versión anterior de la norma, la alta dirección debe demostrar el liderazgo y el compromiso con el sistema de gestión de la calidad, ; lo cual se evidencia con la participación, seguimiento a la política y a los objetivos de calidad, asegurando que sean compatibles con el direccionamiento estratégico y el contexto de la organización, que sean divulgados promoviendo la toma de conciencia, que se apliquen y deben estar orientados a lo que es el negocio de la organización, sin importar que sea una empresa pública, privada con o sin ánimo de lucro, permitiendo los recursos tanto económicos como humanos y promoviendo la mejora continua y la medida de la eficacia del sistema. La alta dirección lo concreta, colocando en el presupuesto financiero el rublo correspondiente al sistema de gestión, firmando la política, dejando actas de seguimiento a los objetivos de calidad.

Con respecto al enfoque hacia los clientes, es nuevo que debe determinar cuáles son los riesgos que puedan afectar la conformidad del producto y la prestación de los servicios, debe asegurar que la organización cumple con los requisitos del cliente, sus especificaciones, los reglamentarios, y los de ley; puede además dejar registro de visitas a ellos, la atención de sus quejas y sugerencias buscando aumentar la satisfacción.

Como aspecto importante de la política de calidad, la nueva versión debe estar disponible para los clientes, proveedores, para todos los miembros de la organización y al entorno [4], debe ser adecuada con el propósito del negocio y debe servir de marco para revisión de los objetivos de calidad; es decir, los objetivos que formule la empresa deben estar enmarcados en la política de calidad siendo coherente con el direccionamiento estratégico y el contexto de la organización.

Sobre los roles y responsabilidades de los cargos que afectan el sistema de gestión de la calidad, se requiere que sean claros y definidos, que los empleados las conozcan y las entiendan para cumplir con el enfoque hacia los clientes, realizar un manual de funciones y responsabilidades, en donde se definen los requisitos de los cargos por educación, experiencia, competencias evidencia el cumplimiento de los requisitos de la norma y la satisfacción de los clientes.

\section{F. Planificación para el sistema de gestión de la calidad}

En este requisito se incluyó la parte del enfoque hacia el riesgo o pensamiento basado en el riesgo y oportunidades y hace referencia a las cuestiones que afectan la organización sea interno o externo que se mostraron en el numeral 4 de la norma; el objetivo es evitar, prevenir, reducir, asumir, mantenerlos efectos indeseados para alcanzar los objetivos de calidad y la satisfacción de los clientes [5] ;estos deben estar planificados implementando las acciones de cómo tratarlos para medir su eficacia. Como se hizo mención en la parte de la compatibilidad con otras normas cada organización puede referenciar, si se quiere, la ISO 31000 sobre la gestión del riesgo, la norma de gestión pública GP 1000 en donde se elabora un mapa de riesgos teniendo en cuenta los siguientes requisitos y que son fundamentales para las organizaciones: elaborar una política empresarial del riesgo en donde se determina el cómo los afronta, los comunica, cumple normas legales vigentes, los reduce los elimina, o convive con ellos, a los objetivos de calidad debe blindarlos mediante el estudio de gestión del riego para poder alcanzar las metas trazadas en el direccionamiento estratégico y en el contexto de la organización. Los riesgos se deben de identificar primeramente, localizando en que proceso se pueden presentar, describiendo su tipo de riesgo, si es económico, ambiental, de salud y seguridad en el trabajo etc., si es interno o externo sea local, regional, nacional, internacional, después se debe hacer un análisis de las causas y consecuencias que puede afectar el negocio y el cumplimiento de los objetivos de calidad, en el análisis se debe asignar la probabilidad, el impacto económico que puede originar, y el nivel de control o detección o prevención que tiene la empresa 
para evitarlo. Con estos insumos se debe hacer la etapa de valoración para la cual existen fórmulas y gráficas que ayudan a dar una prioridad de atención del riesgo; se pueden visualizar en la web del departamento administrativo de la función pública, www.dafp.org. En la valoración se debe especificar si es un riesgo de una acción que ya pasó o que es totalmente prevención, si es catastrófico o inaceptable, si es moderado, tolerable o aceptable a convivir con él. Para finalizar, se debe tomar las acciones de mejora continua, aprovechando las oportunidades, describiendo la estrategia a implementar, el responsable, el tiempo y la meta con indicadores que se busca alcanzar, para eliminar, reducir, obteniendo el riesgo residual que queda después de su administración para la mejora continua.

Los Objetivos de calidad se deben establecer para cada proceso y a los niveles que permitan que se puedan conocer en toda la organización, deben ser coherentes con el direccionamiento estratégico y con la política, ser medibles y que se puedan alcanzar, contar con las metas e indicadores los responsables de administrarlos, con fechas de cumplimiento y se les debe hacer seguimiento ajustándolos cuando se requieran y lo más importante, la estrategia de cómo alcanzarlos , el resultado de los objetivos ; al finalizar los periodos de metas, son el insumo importante para la evaluación de desempeño de los empleados y la planificación para nuevos periodos y si se requiera cambiar, se deben asignar los recursos necesarios de orden económico o humano con nuevas responsabilidades necesarias para cumplir los objetivos planteados.

\section{G. Soporte}

En la versión anterior se denominó gestión de los recursos como requisito; la organización debe asignar los recursos económicos y el personal necesarios para establecer, implementar y mantener el sistema de gestión de la calidad, teniendo en cuenta lo que requerimientos de proveedores externos y de sus limitaciones de acuerdo con su estructura organizacional.

Las condiciones de la infraestructura con que cuenta la organización para realizar sus productos y prestar sus servicios, se debe mantener en óptima condición, se deben establecer los controles y los mecanismos para hacerle seguimiento; con programas de mantenimiento preventivo podemos asegurar el control a edificios, maquinaria y equipos, vehículos, servicios asociados, computadores etc. y cumplir con este requisito.

La organización debe asegurar que las condiciones ambientales no afecten la realización de los productos, como son las condiciones físicas, sociales, ambientales; por ejemplo: la humedad, el ruido, la lluvia, altas o bajas temperaturas. Con un buen programa de inspección se puede asegurar el cumplimiento del requisito.

Los instrumentos de medida utilizados en las máquinas o equipos para verificación del estado de los productos, materiales en proceso etc., se deben mantener en óptimas condiciones y se deben controlar haciendo las verificaciones de su estado de funcionamiento [6], enviándolos a calibrar a intervalos planificados; según las normas se recomienda el tipo de medidor, o teniendo una rutina interna de comparación con patrones adecuados propios protegidos, que sean confiables y que permitan un aseguramiento de calidad de las mediciones.

En la nueva versión, la capacitación se denomina conocimientos organizativos y la institución debe asignar los recursos para actualizar los funcionarios cuando lo requiera; se debe hacer un programa iniciando cada año con las necesidades de capacitación requerida, sea por nuevos proyectos o en busca de la mejora continua del desempeño de las personas producto de las evaluaciones de desempeño.

Como se mencionó en el numeral 5, se debe contar con manuales de funciones y responsabilidades que incluyan los requisitos de los cargos por educación, experiencia y las competencias básicas, las genéricas y técnicas y así asegurar que la organización contrata sus empleados con requisitos establecidos; si se dificulta la contratación, se puede pueden ingresar y capacitar hasta que cumplan el requisito de competencia.

Sobre la toma de conciencia está igual que la versión anterior con una encuesta de clima organizacional que incluya como es la comunicación en ambas direcciones, si la implementación del sistema de calidad ha contribuido a mejorar su desempeño, si conoce los objetivos, si entiende la política de calidad y como es su contribución al sistema de gestión de la calidad; esta encuesta se debe aplicar como mínimo, una vez al año.

La comunicación tanto interna como externa de todo lo que afecta el sistema de gestión, se unieron en esta versión y la organización debe definir el qué, el quién, el cuándo, el contenido de lo que se va a comunicar.

En la versión anterior se tenían 6 procedimientos obligatorios que se debían documentar; ahora no se tienen procedimientos obligatorios, dos de ellos eran el control de documentos y control de registros, los unió y los denominó como información documentada; los contenidos siguen siendo los mismos, así como la determinación de quien está autorizado para crear, modificar, eliminar, actualizar, modificar los documentos de la organización, igualmente quien hace la revisión y la aprobación, que tengan título, la fecha de vigencia, la versión, si están en medio magnético o escrito, que sean legibles sin tachones, y se debe hacer control respectivo de los registros, especificando donde están o quien los maneja, como se pueden recuperar cuando se debe obtener una nueva copia, controlar la distribución de aquellos que son de manejo especial, el tiempo de retención marcando si son vigentes o no y se conservan por necesidades históricas, la disposición final, y a los documentos de origen externo de manejo especial que determine la empresa como se controlan hasta llegar al destinatario. Con dos formatos, uno para los documentos y otro para los registros, se puede asegurar el requisito de la información documentada.

\section{H. Operación}

La operación es el nuevo nombre de lo que se denominaba la realización del producto en el numeral 7 de la versión 2008; lo 
nuevo es que, en la planificación y control operacional, se deben tener en cuenta las acciones para administrar el riesgo como se describió en el numeral 4 y 6 de esta nueva versión, estableciendo los criterios para aceptar los productos y servicios y los cambios se deben realizar de una manera planificada, tomando las acciones para mitigar los efectos adversos. En las evidencias este numeral se debe aprovechar los procedimientos o instructivos elaborados y agregarles los puntos de control que se deben tener desde los elementos de entrada del proceso hasta los de su salida.

La organización debe determinar los requisitos para los productos y servicios que presta, estableciendo procesos de comunicación con los clientes, para garantizar que sus productos y servicios son entregados como lo solicitaron, que hay canales de comunicación para hacer sugerencias, reclamos , quejas para la mejora continua, que los requisitos deben estar muy claros, completos, escritos en los catálogos o en los contratos que firman con sus clientes, que incluyan primeramente los requisitos de ley y reglamentarios; estos requisitos se deben revisar para asegurar que son vigentes, que la ley o reglamentos no han cambiado, ni las especificaciones técnicas de los productos fabricados o los servicios prestados; esto se debe hacer durante periodos planificados y dejar los registros correspondientes.

Cuando la organización hace diseño y desarrollo de productos o servicios, debe establecer un proceso para realizar, implementar, y mantenerlos; para lo cual, planifica todas las actividades que incluyan los elementos de entrada [7], como encuestas o estudios de mercado que permitan justificar un producto nuevo o servicio, o el desarrollo o modificación de estos, los requisitos legales, reglamentarios y normativos que se deben cumplir y especificar las consecuencias por los fracasos que se puedan presentar; la planificación debe incluir además, el control que se debe hacer desde que se inician las actividades hasta la salida, revisando en todas las actividades o pasos en la producción o en la prestación del servicio que requieran control, ya sea con equipos como balanzas, metros, dinamómetros, manómetros etc., o medidas y lecturas de supervisión que se puedan registrar para garantizar que se está cumpliendo con los requisitos especificados en la planificación. La norma exige que se deben hacer la verificación especificando los requisitos de entrada del cliente y que compare en la salida, si se cumple con lo que se determinó en el contrato o en la solicitud de pedido. Como requisito para entender si lo que realiza una empresa es diseño y desarrollo o es mejora continua, debe existir la validación por parte del cliente si lo que él solicito, es lo que se entregó en la salida, si no existe un cliente que valide es mejora continua, si durante las etapas de fabricación o prestar el servicio se cambia lo planificado; se deben dejar los registros de los elementos cambiados.

En cuanto a compras se refiere en la anterior versión, ahora se llama control de los productos y servicios suministrados externamente y la organización debe asegurar que se cumplan con los requisitos de los clientes, haciendo una selección de sus proveedores mediante la evaluación de su infraestructura que cumpla con las especificaciones técnicas, hacer seguimiento de su desempeño, y mínimo cada año hacer la reevaluación teniendo en cuenta el cumplimiento en calidad, cantidad, servicio posventa etc., debe dejar el registro para evidencia, teniendo en cuenta que se les debió dar toda la información necesaria para su producción o prestación del servicio, como los requisitos del cliente, o especificaciones técnicas, las unidades, tiempos de entrega, cantidades, requisitos de calidad, competencias del personal que se requiere emplear, el control que es necesario efectuar y, antes de entregar a los clientes la organización, debe realizar la verificación de esos productos y servicios dejando los registros pertinentes.

Para el control de la producción y de la prestación del servicio, la organización debe tener definidos los parámetros físicos y los controles que debe realizar durante la fabricación y el servicio, en que etapas, con que métodos y equipos aseguran las pruebas y cumplir con los elementos de salida de los procesos y con los requisitos de los clientes; cuando son pruebas cualitativas o no existen instrumentos para asegurar las pruebas, se deben validar los resultados con base en la experiencia de la organización, del profesionalismo de sus empleados, de las normas y documentos empleados para respaldar dichos resultados.

Para facilitar la identificación y trazabilidad de los productos y servicios durante su producción y la prestación del servicio, la organización debe implementar los medios adecuados para cuando se requiera investigar las quejas, los reclamos de los clientes o por necesidad de la empresa; esto puede ser con códigos de barras, de radio frecuencia, la identificación de los resultados del aseguramiento de la calidad con registros de confiable adquisición.

Cuando la organización tiene en su espacio equipos, materiales, materias primas, herramientas, datos personales, etc., que son propiedad de los clientes o proveedores externos, debe identificarlos, y asegurar que no se deterioren y los debe mantener como si fueran de su propiedad; en el caso que se presente deterioros, daños en ellos se debe avisar al cliente o proveedor.

Durante el transporte, almacenaje, manipulación, embalaje de los productos y servicios, la organización debe garantizar la preservación de estos, para asegurar las entregas a los clientes cumpliendo con los requisitos pactados, esto incluye las actividades posteriores a la entrega, se debe informar al cliente de los cuidados a tener, de los riesgos del producto y los servicios, los requisitos legales, reglamentarios y sobre la garantía que cubre, y obligaciones contractuales, como mantenerlo, de la disposición final de sus residuos o como reciclarlo.

Si durante la producción y la prestación del servicio, surgen cambios no planificados, la organización debe registrar y hacer control sobre estos cambios, haciendo la revisión para asegurar que se sigue cumpliendo con los requisitos establecidos.

Hasta que no se lleven a cabo todos los controles planificados de los productos y la prestación del servicio con resultados satisfactorios, se debe hacer la liberación de los productos y 
servicios, la organización debe establecer las personas responsables de esta etapa y quedar los registros correspondientes.

En la versión anterior de la norma, lo que se denominado control del producto no conforme se llama ahora como control de los elementos de salida del proceso, los productos y los servicios no conformes. Durante toda su fabricación, la organización debe identificar lo no conforme, separar o identificar con letreros en lugares separados para evitar el uso mal intencionado o por error de los que no es conforme, lo no conforme se puede producir y enviar al cliente solo con autorización dejando los registros correspondientes.

\section{Evaluación de desempeño}

Lo que no se mide no se puede mejorar; la organización debe definir qué es lo necesario para hacer seguimiento que le genera valor agregado, se debe especificar la metodología, el análisis, de acuerdo con los requisitos especificados del producto o el servicio y por lo menos una vez al año se debe hacer una medida de la satisfacción del cliente, puede ser con encuestas, llamadas, buzones de sugerencia etc. Las respuestas se deben analizar y evaluar y se deben formular los objetivos para la mejora continua y aumento de su satisfacción.

Las auditorías internas se deben programar a intervalos planificados, como mínimo una vez por año y deben cubrir todos los elementos de la norma; como buena práctica deberían realizarse durante todo el año, con auditores internos capacitados y con las competencias y metodología requerida según la norma ISO 9011, los resultados deben ser informados a la dirección y se deben tomar las correcciones y acciones correctivas necesarias de los hallazgos encontrados.

La Alta dirección debe a intervalos planificados hacer la revisión por la dirección, que incluya el estado de revisiones anteriores, de las auditorias, el desempeño de los proveedores externos y el personal, el estado de las no conformidades y acciones correctivas, de la administración del riesgo y las oportunidades, del resultado de auditorías internas y externas, de la satisfacción del cliente, de los productos y servicios no conformes, de las quejas, reclamos de los clientes y que decisiones se tomaron.

\section{J. Mejora}

La organización debe proponer en sus objetivos la mejora de los procesos. Los productos y servicios, y todo lo que afecte el sistema de gestión de la calidad, las no conformidades encontradas, se deben evaluar y analizar y hacer propuestas de eliminarlas, planificando el cuándo y el cómo se va actuar; primero se debe hacer las correcciones inmediatas y toda no conformidad o hallazgo que por su impacto en la organización afecto el sistema de gestión, se deben hacer las acciones correctivas necesarias para evitar que se vuelvan a presentar, haciendo el análisis de las causas, su impacto y las propuestas de mejora continua por los procesos involucrados. Para esto se pueden aplicar las herramientas estadísticas como análisis de Pareto, diagrama de causa y efecto, análisis de la causa raíz, los 5 porqués, análisis de regresión etc.

\section{CONCLUSIONES}

Sobre la nueva versión de la norma ISO 9001:2015, podemos concluir que se le dio mucho énfasis al direccionamiento estratégico de las organizaciones, las cuales deben fijar su política de calidad de acuerdo con la misión de la institución y a todo lo que quede incluido en la política se le deben fijar objetivos para facilitar el cumplimiento de su visión para el corto, mediano y largo plazo que sean coherentes con el propósito de aumentar la satisfacción del cliente.

La filosofía de la nueva versión está fundamentada en el pensamiento basado en el riesgo y obliga a que se identifiquen todos los riesgos que pueden afectar el cumplimiento de los objetivos de calidad de la institución. Para identificar estos riegos se debe tener en cuenta el entorno local, regional, nacional e internacional y los factores económicos, tecnológicos, culturales, sociales, mercado, legales, etc.

La nueva norma reordenó los requisitos, agrupándolos de acuerdo con el planear, hacer, verificar y actuar, en los diferentes numerales del 0 al 10 ; esta estructura queda obligatoria para todas las normas de sistemas de gestión existentes (ISO 14001, ISO 45001, etc.)

En la versión de la norma ISO 9001:2008, era obligatorio documentar 6 procedimientos y tener un manual de calidad; en esta nueva se eliminó ese requisito.

Para las organizaciones que tienen la norma ISO 9001:2008, se recomienda no eliminar la documentación existente, la deben adecuar a la nueva versión, dejando la estructura de las manuales que facilitan su implementación.

\section{REFERENCES}

[1] Instituto Colombiano de Normas Técnicas ICONTEC, Norma Técnica Internacional ISO 9001: 2015, Sistema de Gestión de la Calidad, requisitos, Objeto y campo de aplicación, p.9, 2015

[2] Instituto Colombiano de Normas Técnicas ICONTEC, Norma Técnica Internacional ISO 9001: 2015, Sistema de Gestión de la Calidad, requisitos, Términos y definiciones, p. 9, 2015

[3] Instituto Colombiano de Normas Técnicas ICONTEC, Norma Técnica Internacional ISO 9001: 2015, Sistema de Gestión de la Calidad, requisitos, Contexto de la organización, pp. 22-24, 2015

[4] Instituto Colombiano de Normas Técnicas ICONTEC, Norma Técnica Internacional ISO 9001: 2015, Sistema de Gestión de la Calidad, requisitos, Liderazgo, pp. 24-26, 2015

[5] Instituto Colombiano de Normas Técnicas ICONTEC, Norma Técnica Internacional ISO 9001: 2015, Sistema de Gestión de la Calidad, requisitos, planificación para el sistema de gestión de calidad, pp. 26-27

[6] Instituto Colombiano de Normas Técnicas ICONTEC, Norma Técnica Internacional ISO 9001: 2015, Sistema de Gestión de la Calidad, requisitos, operación, pp. 30-37

[7] Instituto Colombiano de Normas Técnicas ICONTEC, Norma Técnica Internacional ISO 9001: 2015, Sistema de Gestión de la Calidad, requisitos, evaluación del desempeño, pp. 37- 40, 2015 


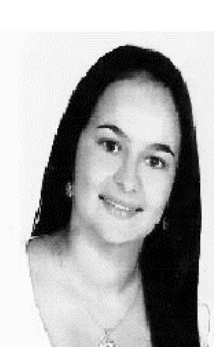

Ángela María Buritica Macías, Ingeniera Industrial, Magister en Administración del desarrollo humano y organizacional de la Universidad Tecnológica de Pereira, Docente Facultad de Ciencias Empresariales en el área de administración de la Universidad Tecnológica de Pereira desde 2010, Participante en grupo Investigación en calidad Y administración de la Facultad de Ciencias empresariales de la Universidad Tecnológica de Pereira. Consultora en sistemas de Gestión de calidad y recursos humanos desde el año 2004.

ORCID: http://orcid.org/0000-0003-0417-6153

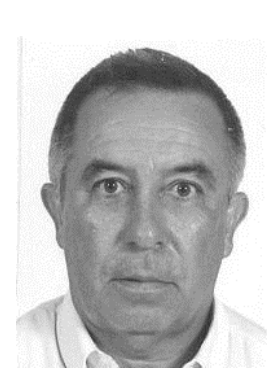

Carlos Alberto Buriticá Noreña, Tecnologo Electricista de la Universidad Tecnológica de Pereira en 1974, Ingeniero electricista de la Universidad Tecnológica de Pereira en el año 1980, Magister en Administración Económica y Financiera de la Universidad Tecnológica de Pereira año 1992. Docente facultad de Ciencias Empresariales, autor de tres libros manufactura flexible, Caracterización y propuesta de producción limpia en los principales procesos productivos regionales y aplicación de modelos en la producción, Autor de varios artículos en el área de calidad y normalizan técnica. Director del grupo de investigación de manufactura flexible y del grupo de investigación de calidad y normalización técnica hasta año 2016, miembro activo de ambos grupos. Consultor en gestión de calidad y normalización tecnica.

ORCID:http://orcid.org/0000-0002-8910-3000

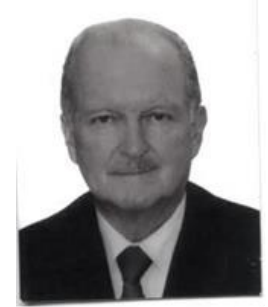

José Germán López Quintero, Ingeniero Electricista, Universidad Tecnológica de Pereira, 2 de marzo de1973

Profesor Titular de Ingeniería Eléctrica de la Universidad Tecnológica de Pereira

Vicerrector Académico, 2000 - 2010, de la Universidad Tecnológica de Pereira

Vicerrector de Investigaciones, Innovación y Extensión, 2010 - 2014, de la Universidad Tecnológica de Pereira. Rector (encargado) de la Universidad Tecnológica de Pereira. Director Ingeniería Eléctrica desde el año 2015 a la fecha, de la Universidad Tecnológica de Pereira Ha publicado varios libros y artículos en revistas especializadas nacionales.

ORCID: http://orcid.org/0000-0001-5931-1397 